\title{
Evaluation of prostaglandin D2 as a CSF leak marker: implications in safe epidural anesthesia
}

\author{
This article was published in the following Dove Press journal: \\ Local and Regional Anesthesia \\ I July 201 I \\ Number of times this article has been viewed
}

\section{Sirish Kondabolu \\ Rishimani Adsumelli \\ Joy Schabel \\ Peter Glass \\ Srinivas Pentyala}

Department of Anesthesiology,

School of Medicine, Stony

Brook Medical Center,

Stony Brook, New York, USA
Correspondence: Srinivas Pentyala Department of Anesthesiology, HSC L4-070, Stony Brook Medical Center, Stony Brook, NY, I I 794-8480, USA

Tel + I 63 I 4442974

Fax + I 63| 4442907

Email srinivas.pentyala@stonybrook.edu
Background: It is accepted that there is a severe risk of dural puncture in epidural anesthesia. Of major concern to anesthesiologists is unintentional spinal block. Reliable identification of cerebrospinal fluid (CSF) from the aspirate is crucial for safe epidural anesthesia. The aim of this study was to determine whether prostaglandin D2 could be clinically used as a marker for the detection of CSF traces.

Methods: After obtaining Institutional Review Board approval and patient consent, CSF was obtained from patients undergoing spinal anesthesia, and blood, urine, and saliva were obtained from normal subjects and analyzed for prostaglandin D2 (PGD). CSF ( $n=5$ ) samples were diluted with local anesthetic (bupivacaine), normal saline and blood in the ratios of 1:5 and 1:10. PGD levels in the CSF samples were analyzed with a PGD-Methoxime (MOX) EIA Kit (Cayman Chemicals, MI). This assay is based on the conversion of PGD to a stable derivative, which is analyzed with antiserum specific for PGD-MOX.

Results: Different concentrations of pure PGD-MOX conjugate were analyzed by EIA and a standard curve was derived. PGD levels in CSF and CSF with diluents were determined and the values were extrapolated onto the standard curve. Our results show a well-defined correlation for the presence of PGD both in straight CSF samples and in diluted CSF (dilution factor of 1:5 and 1:10).

Conclusion: Prostaglandin D2 was reliably identified in CSF by enzyme-linked immunosorbent assay when diluted with local anesthetic, saline, and serum, and can be used as a marker to identify the presence of CSF in epidural aspirates.

Keywords: epidural, cerebrospinal fluid, leak, marker, prostaglandin D2

\section{Introduction}

Unintentional spinal block occurs if the medication intended to be placed in the epidural space enters the intrathecal space. ${ }^{1}$ This is a serious complication that requires immediate diagnosis and prompt cardiopulmonary resuscitation. ${ }^{2,3}$ In the obstetric population, this can lead to severe fetal compromise necessitating immediate cesarean section. In a retrospective study of maternal mortality in 1979-2002, death due to anesthesia was found to be the sixth leading cause of pregnancy-related mortality in the US, and 9\% of these deaths were attributed to high spinal blocks following epidural anesthesia. ${ }^{4}$

Currently none of the clinical tests used can reliably differentiate cerebrospinal fluid (CSF) from other substances in all situations. Chemical analysis of fluid for glucose and protein is unreliable as a means of determining if the fluid is CSF. ${ }^{5,6}$ Radiographic studies, with or without intrathecal injection of dye or radioisotope, are not always successful in demonstrating small or delayed CSF leaks, and are technically difficult and time-consuming. At present, electrophoresis of fluid from a suspected CSF leak 
combined with immunofixation to detect beta-2 transferrin, a unique CSF protein, is a highly specific and widely used method of detecting $\mathrm{CSF}^{7-9}$ However, this technique is cumbersome, time-consuming, and very expensive, with few clinical laboratories being able to perform this test.

Recent studies have revealed that prostaglandin D2 (PGD), an endogenous sleep-promoting substance, accumulates in CSF. ${ }^{10-12}$ Prostaglandin D2 synthase in the brain catalyzes the isomerization of prostaglandin $\mathrm{H} 2$ to $\mathrm{PGD}$, which circulates in the CSF. ${ }^{13}$ Although PGD is present in other body fluids, due to the presence of high levels in CSF, PGD could be a valuable tool as a marker for the detection of CSF traces. The aim of this study was to determine whether a specific CSF marker like PGD has any predictive value when CSF is diluted with local anesthetic, saline, and serum. An in vitro study was performed to identify PGD in CSF when diluted with local anesthetic, saline, or serum in a 1:5 and 1:10 dilution. Using an enzymelinked immunosorbent assay, PGD was reliably identified in all CSF samples independent of dilution or diluent. These results will aid in the development of a detection kit, which will be beneficial for clinicians requiring a rapid and reliable bedside test to detect CSF leak.

\section{Methods and materials}

After obtaining institutional review board approval and patient consent, CSF samples were obtained from five healthy patients undergoing spinal anesthesia in the labor and delivery unit of University Hospital and analyzed for PGD. CSF samples diluted with various volumes of saline, local anesthetic, and serum were analyzed for the presence of PGD. Urine and saliva samples were also analyzed for evaluation of the relative PGD amounts. The assay was performed by enzyme-linked immunosorbent assay (Cayman Chemicals, Ann Arbor, MI). The results were then correlated and checked for their specificity. All chemicals used were purchased from Sigma Chemicals (St Louis, MO).

In this assay, CSF samples were diluted with local anesthetic (bupivacaine), normal saline, and serum in the ratios of 1:5 and 1:10. PGD levels in the straight and diluted CSF samples were analyzed using a PGD-methoxime enzymelinked immunosorbent assay kit (Cayman Chemicals). This enzyme-linked immunosorbent assay is based on the conversion of PGD to a stable methoxime derivative, where treatment of PGD in CSF with methoxime hydrochloride will convert PGD into PGD-methoxime. This stable conjugate is analyzed with rabbit antiserum binding sites specific for PGD-methoxime in the enzyme-linked immunosorbent assay. Because the concentration of the PGD-methoxime tracer is held constant while the concentration of PGD-methoxime varies, the amount of PGD-methoxime tracer that is able to bind to the rabbit antiserum will be inversely proportional to the concentration of PGD-methoxime in the well. This rabbit antiserum PGD-methoxime (either free or tracer) complex binds to the mouse monoclonal antirabbit IgG that has been previously attached to the well. The plate is washed to remove any unbound reagents and then Ellman's reagent (which contains the substrate to AChE) is added to the well. The product of this enzymatic reaction has a distinct yellow color and absorbs strongly at $412 \mathrm{~nm}$. The intensity of this color, determined spectrophotometrically, is proportional to the amount of PGD-methoxime tracer bound to the well, which is inversely proportional to the amount of free PGDmethoxime present in the well during the incubation.

\section{Results}

CSF samples obtained from five healthy patients undergoing spinal anesthesia in the labor and delivery unit of University Hospital were diluted in different ratios with saline and with different concentrations of drugs and analyzed for PGD. To evaluate the relative concentrations of PGD in CSF samples, initially different concentrations of pure PGD-methoxime conjugate (see Methods section) were analyzed by methoxime and a standard curve was derived (Figure 1). PGD in CSF and CSF with diluents in different ratios was determined using a PGD-methoxime enzymelinked immunosorbent assay protocol and the values were extrapolated onto the standard curve. Our results show a well defined correlation for the presence of PGD in CSF samples. The PGD marker was also easily identified in CSF samples diluted with different eluates (Figure 2). The fact that PGD

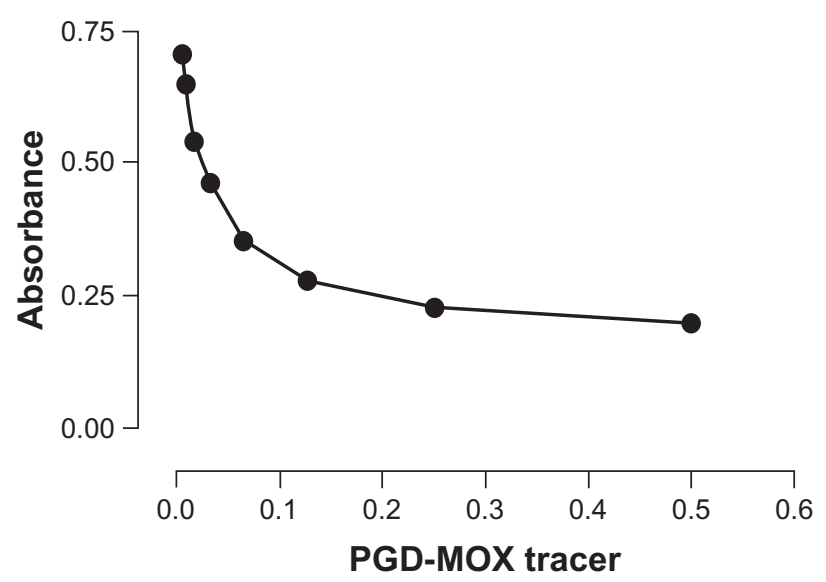

Figure I Standard curve of PGD amounts derived by analyzing with PGD-MOX enzyme linked immunoabsorbant assay.

Abbreviations: PGD, prostaglandin D2; MOX, methoxime. 


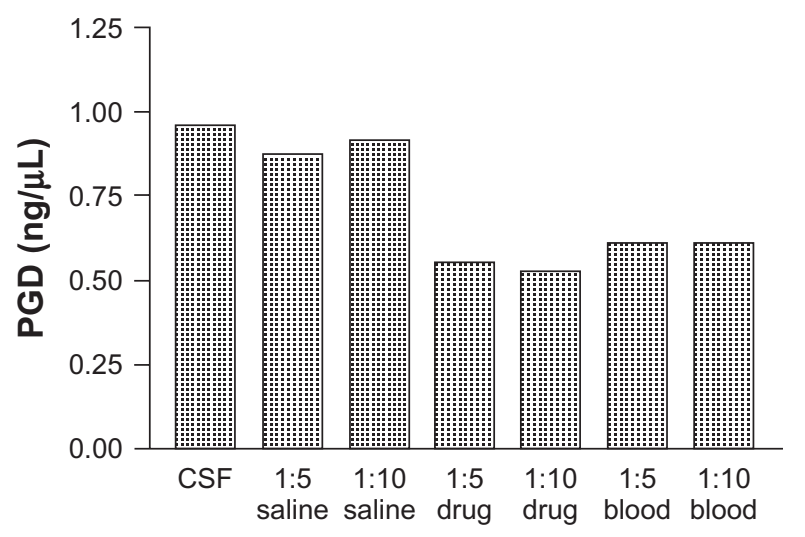

Figure 2 CSF samples were diluted with local anesthetic (bupivacaine), normal saline and blood in the ratios of I:5 and I:I0. PGD levels in the straight and diluted CSF samples were analyzed with a PGD2-Methoxime (MOX) EIA Kit (Cayman Chemicals, MI).

Abbreviations: PGD, prostaglandin D2; CSF, cerebrospinal fluid.

was detected in such small amounts of CSF shows that this test is highly sensitive.

\section{Discussion}

In this in vitro analysis, PGD was identified in all the CSF samples. During the epidural procedure when the dura is punctured with an epidural needle there is usually free flow of CSF. However, when saline is used for loss of resistance there may be a need to use a test that confirms the presence of CSF. The various physicochemical bedside tests that are currently used to detect the presence of CSF in this situation, ie, rate of flow, temperature, tests for glucose and protein, and $\mathrm{pH}$, cannot provide reliable identification. ${ }^{5,14,15}$ When fluid is aspirated from the catheter, even before contamination with local anesthetic, a combination of tests like glucose, protein, and $\mathrm{pH}$ may be used. However, contamination with blood and interstitial fluid can result in positive glucose and protein, as well as a change in $\mathrm{pH}$ close to that of CSF which may lead to false positive identification of CSF and needless repetition of epidural procedures. ${ }^{6}$ When the epidural aspirate contains local anesthetic, a thiopental precipitation test will form precipitate with local anesthetic but not with CSF. This test was used in the past to differentiate reliably between local anesthetic and CSF. ${ }^{6,16}$ Recently, this test has proven to be unreliable with the current change of practice whereby a mixture of dilute local anesthetics and opioids are administered and both false positive and false negative results have been noted, highlighting the potential for serious complications. ${ }^{6,16}$ The issue is even more complex when the aspirate is obtained during the course of continuous epidural anesthesia. In this situation, the aspirate is a mixture of local anesthetic, opioid, interstitial fluid, blood, and possibly CSF. Thus, the bedside test available at present can be misleading. The standard test dose with $1.5 \%$ lidocaine and epinephrine $5 \mu \mathrm{g} / \mathrm{cc}$ is routinely used to detect subarachnoid placement by producing clinically detectable sensory and motor blockade that is not unsafe for patients. However, this test dose can be dangerous and difficult to interpret in patients with pre-existing partial block. Furthermore, in parturients, it can produce a dangerously high block requiring aggressive treatment. Richardson et al in their report of high block following a test dose in five parturients raise the question of its safety. ${ }^{17}$

In our study using the enzyme-linked immunosorbent assay technique, PGD was reliably identified in CSF when diluted with local anesthetic, saline, and serum. Although PGD was reported to be present in other biological fluids, our results showed no significant PGD in urine and saliva samples when compared with high levels of this marker in CSF. When analyzed by the PGD-methoxime enzymelinked immunosorbent assay protocol, PGD levels were found to be lower in CSF diluted with either serum or local anesthetic, suggesting that serum factors and bupivacaine interfere with the spectrophotometric analysis. However, the enzyme-linked immunosorbent assay method was able to identify PGD in these diluted samples, also suggesting that this test can be used effectively to determine CSF leaks when admixed with different entities, like drugs and serum. This PGD determination test would help to identify even minute traces of CSF leaks. Detection of PGD, particularly in epidural eluates, will be of immense value in indicating a potential CSF leak to the anesthesiologist. Through the development of a rapid detection technique to detect PGD, it can be anticipated that risks in epidural procedures can be minimized.

\section{Conclusion}

There are many complications associated with epidural anesthesia. In order to reduce serious complications, such as unintentional spinal blockade, and to ensure safe epidural procedures, a rapid detection method is necessary to detect CSF in epidural aspirate. In this in vitro study, a PGD assay reliably identified CSF. Although this kit is not yet clinically available as a bedside test, the possibility of development of such test in the near future is very likely. Furthermore, such a test is of great value not only for anesthesiologists but also in other instances where fluid needs to be identified as CSF.

\section{Disclosure}

The authors report no conflicts of interest in this work. 


\section{References}

1. Agarwal D, Mohta M, Tyagi A, Sethi AK. Subdural block and the anaesthetist. Anaesth Intensive Care. 2011;38:20-26.

2. Mullanu C, Gaillat F, Scemama F, Thibault S, Lavand'homme P, Auffray JP. Acute toxicity of local anesthetic ropivacaine and mepivacaine during a combined lumbar plexus and sciatic block for hip surgery. Acta Anaesthesiol Belg. 2002;53:221-223.

3. Lee PK, Kim JM. Lumbar epidural blocks: a case report of a life-threatening complication. Arch Phys Med Rehabil. 2000;81:1587-1590.

4. Hawkins JL, Chang J, Palmer SK, Gibbs CP, Callaghan WM. Anesthesia-related maternal mortality in the United States: 1979-2002. Obstet Gynecol. 2011;117:69-74.

5. Chan DT, Poon WS, Ip CP, Chiu PW, Goh KY. How useful is glucose detection in diagnosing cerebrospinal fluid leak? The rational use of CT and beta-2 transferrin assay in detection of cerebrospinal fluid fistula. Asian J Surg. 2004;27:39-42.

6. Waters JH, Rizzo VL, Ramanathan S. A re-evaluation of the ability of thiopental to identify cerebrospinal fluid in epidural catheter aspirate. J Clin Anesth. 1995;7:224-227.

7. Sampaio MH, de Barros-Mazon S, Sakano E, Chone CT. Predictability of quantification of beta-trace protein for diagnosis of cerebrospinal fluid leak: cutoff determination in nasal fluids with two control groups. Am J Rhinol Allergy. 2009;23:585-590.

8. Ryall RG, Peacock MK, Simpson DA. Usefulness of beta 2-transferrin assay in the detection of cerebrospinal fluid leaks following head injury. J Neurosurg. 1992;77:737-739.
9. Meurman OH, Irjala K, Suonpaa J, Laurent B. A new method for the identification of cerebrospinal fluid leakage. Acta Otolaryngol. 1979;87:366-369.

10. Hayaishi O. Molecular mechanisms of sleep-wake regulation: a role of prostaglandin D2. Philos Trans R Soc Lond B Biol Sci. 2000; 355:275-280.

11. Urade Y, Hayaishi O. Prostaglandin D2 and sleep regulation. Biochim Biophys Acta. 1999;1436:606-615.

12. Hayaishi O, Matsumura H. Prostaglandins and sleep. Adv Neuroimmunol. 1995;5:211-216.

13. Urade Y, Hayaishi O. Prostaglandin D synthase: structure and function. Vitam Horm. 2000;58:89-120.

14. Tessler MJ, Wiesel S, Wahba RM, Quance DR. A comparison of simple identification tests to distinguish cerebrospinal fluid from local anaesthetic solution. Anaesthesia. 1994;49:821-822.

15. el-Behesy BA, James D, Koh KF, Hirsch N, Yentis SM. Distinguishing cerebrospinal fluid from saline used to identify the extradural space. $\mathrm{Br}$ J Anaesth. 1996;77:784-785.

16. Lowenwirt I, Axelrod F, Krishnamurthy U. Failure of the thiopental precipitation test to detect cerebrospinal fluid after dural puncture with current epidural solutions for labor analgesia. Reg Anesth. 1995;20:459-460.

17. Richardson MG, Lee AC, Wissler RN. High spinal anesthesia after epidural test dose administration in five obstetric patients. Reg Anesth. 1996;21:119-123.

\section{Publish your work in this journal}

Local and Regional Anesthesia is an international, peer-reviewed, open access journal publishing on the development, pharmacology, delivery and targeting and clinical use of local and regional anesthetics and analgesics. The journal welcomes submitted papers covering original research, basic science, clinical studies, reviews \& evaluations,

\section{Dovepress}

guidelines, expert opinion and commentary, case reports and extended reports. The manuscript management system is completely online and includes a very quick and fair peer-review system, which is all easy to use. Visit http://www.dovepress.com/testimonials.php to read real quotes from published authors. 